Event-by-Event Simulation of Induced Fission

R. Vogt, J. Randrup

December 26, 2007

CNR'07

Fish Camp, CA, United States

October 22, 2007 through October 26, 2007 
This document was prepared as an account of work sponsored by an agency of the United States government. Neither the United States government nor Lawrence Livermore National Security, LLC, nor any of their employees makes any warranty, expressed or implied, or assumes any legal liability or responsibility for the accuracy, completeness, or usefulness of any information, apparatus, product, or process disclosed, or represents that its use would not infringe privately owned rights. Reference herein to any specific commercial product, process, or service by trade name, trademark, manufacturer, or otherwise does not necessarily constitute or imply its endorsement, recommendation, or favoring by the United States government or Lawrence Livermore National Security, LLC. The views and opinions of authors expressed herein do not necessarily state or reflect those of the United States government or Lawrence Livermore National Security, LLC, and shall not be used for advertising or product endorsement purposes. 


\title{
Event-by-Event Simulation of Induced Fission
}

\author{
Ramona Vogt*, and Jørgen Randrup ${ }^{* *}$ \\ ${ }^{*}$ Lawrence Livermore National Laboratory, Livermore, CA, USA \\ ${ }^{\dagger}$ Physics Department, University of California at Davis, Davis, CA, USA \\ ${ }^{* *}$ Lawrence Berkeley National Laboratory, Berkeley, CA, USA
}

\begin{abstract}
We are developing a novel code that treats induced fission by statistical (or Monte-Carlo) simulation of individual decay chains. After its initial excitation, the fissionable compound nucleus may either deexcite by evaporation or undergo binary fission into a large number of fission channels each with different energetics involving both energy dissipation and deformed scission prefragments. After separation and Coulomb acceleration, each fission fragment undergoes a succession of individual (neutron) evaporations, leading to two bound but still excited fission products (that may further decay electromagnetically and, ultimately, weakly), as well as typically several neutrons. (The inclusion of other possible ejectiles is planned.) This kind of approach makes it possible to study more detailed observables than could be addressed with previous treatments which have tended to focus on average quantities. In particular, any type of correlation observable can readily be extracted from a generated set of events. With a view towards making the code practically useful in a variety of applications, emphasis is being put on making it numerically efficient so that large event samples can be generated quickly. In its present form, the code can generate one million full events in about 12 seconds on a MacBook laptop computer. The development of this qualitatively new tool is still at an early stage and quantitative reproduction of existing data should not be expected until a number of detailed refinement have been implemented.
\end{abstract}

Keywords: fission simulation

PACS: $24.75 .+\mathrm{i}, 25.85 . \mathrm{Ec}$

\section{INTRODUCTION}

Although nuclear fission has been studied for a long time, this process is still not very well understood. For example, while the average neutron multiplicity per fission event is well known as a function of the initial excitation energy, particularly for major actinides, the neutron spectra are not as well known, especially for more energetic neutrons. The available experimental data tend to be rather inclusive and do not provide detailed insight into the process. For example, the correlation between neutron multiplicity and neutron energy is typically unavailable. Our understanding of fission could likely be advanced by more exclusive data, such as those that could be obtained with a fission time-projection chamber, as has recently been proposed [1].

For the purpose of establishing a convenient tool for theoretical studies of fission phenomena, we are developing a computational model for the complete fission event, starting from the initial agitation of the fissile nucleus, through its subsequent split into two excited fission fragments and their sequential decay chains, ending with the two product nuclei as well as the neutral and charged ejectiles. To permit consideration of correlation observables, the model is implemented as an event generator which provides a sample of complete final states, analogous to approaches used for high-energy nuclear reactions. This method allows the extraction of any desired observable.
We expect our code, FREYA (Fission Reaction Event Yield Algorithm). to be a useful tool for basic research on fission physics. In addition, we anticipate that FREYA will have a number of applications such as homeland security, reactor physics, and transport calculations in stellar environments. For these latter applications in particular, fast event generation is essential. Thus we pay special attention to this requirement.

In the development of the model, we invoke existing data as far as possible. However, as pointed out above, since these data are generally less detailed than required for our purposes, we need to supplement the data with theory. While the associated uncertainty can to some degree be alleviated by adjusting the input parameters to match existing data, our approach naturally identifies future measurements that may yield particular insight and help to reduce the uncertainties, thus improving the practical utiliyy of the code.

At this present early stage, FREYA models neutroninduced fission and the subsequent sequential evaporation of fission neutrons from the fully accelerated fragments. We are in the process of including pre-fission neutron emission at energies of a few $\mathrm{MeV}\left(n^{\text {th }}\right.$-chance fission). Furthermore, we anticipate extensions to spontaneous fission and fission induced by other projectiles as well as gamma and charged particle ejectiles in the final state. Each fission event with FREYA conserves energy, momentum, baryon number and charge while presently ignoring angular momentum. 


\section{METHOD}

FREYA is designed in a modular fashion in order to facilitate continual development as improved data or refined theoretical ideas appear. The simulation is organized to follow the temporal sequence of the fission process from start to finish.

At the start, a neutron with a specified kinetic energy $\varepsilon_{n}$ is absorbed by a fissile nucleus to form the compound nucleus ${ }^{A} Z$ with a certain excitation energy. In its present simple form, where no pre-fission neutron emission is considered, the first process to treat is the fission itself: the split of the mother nucleus ${ }^{A} Z$ into a heavy fragment ${ }^{A_{H}} Z_{H}$ and a light fragment ${ }^{A_{L}} Z_{L}$. In addition to the selection of the specific fission channel, the momenta of the two fragments as well as their resepctive excitation energies must also be selected.

Combined yields of fission fragment mass and totalfragment kinetic energy (TKE) are available for some actinides over part of the incident neutron energy range. We wish to ensure that our treatment reproduces these data. For the time being, the mass partition is selected on the basis of various alternative parametrized probability distributions that depend on the initial excitation energy. The charge partition is subsequently selected from a relatively narrow distribution that conserves the $Z / A$ ratio of the mother nucleus on average.

Once the specific partition has been selected, a tentative value for the total fragment kinetic energy is determined on the basis of a simple model for the scission configuration that does not yet include shell effects. It is important to improve the treatment of the scission configuration by invoking more refined models [2,3].

With the tentative value of TKE determined, the corresponding value of the combined excitation energy can be determined by simple energy conservation. The actual fragment excitation energies are then selected by sampling the associated thermal distribution of the fluctuations in the heat partition. Finally, relativistic kinematics is invoked to determine the actual values of the fragment momenta in a manner that guarantees exact conservation of both momentum and energy.

After the fission fragments have been characterized, the subsequent neutron-evaporation chains are simulated for each fragment separately. At each step, the direction of emission is assumed to be isotropic in the frame of the emitting nucleus and the neutron kinetic energy is selected from the appropriate spectral distribution, taking recoil effects into account to conserve four momentum. The evaporation procedure is iterated as long as the associated decay channel remains open. All emitted particles, as well as the two residual product nuclei (which remain excited but stable with respect to prompt emission), are boosted to the laboratory frame.

\section{PRELIMINARY RESULTS}

In this section, we illustrate some preliminary results with FREYA for $0.53 \mathrm{MeV}$ neutrons bombarding a ${ }^{235} \mathrm{U}$ target.

First, as an example of a rather inclusive observable, we consider the neutron multiplicity distribution. Figure 1 compares our preliminary results with the 1956 thermal neutron data of Diven et al. [4]. The agreement of FREYA with the data is rather good although the average multiplicity from FREYA $(m=2.37)$ is somewhat smaller than that of the data $(m=2.47)$. The agreement with the data may well improve when our planned refinements are introduced.

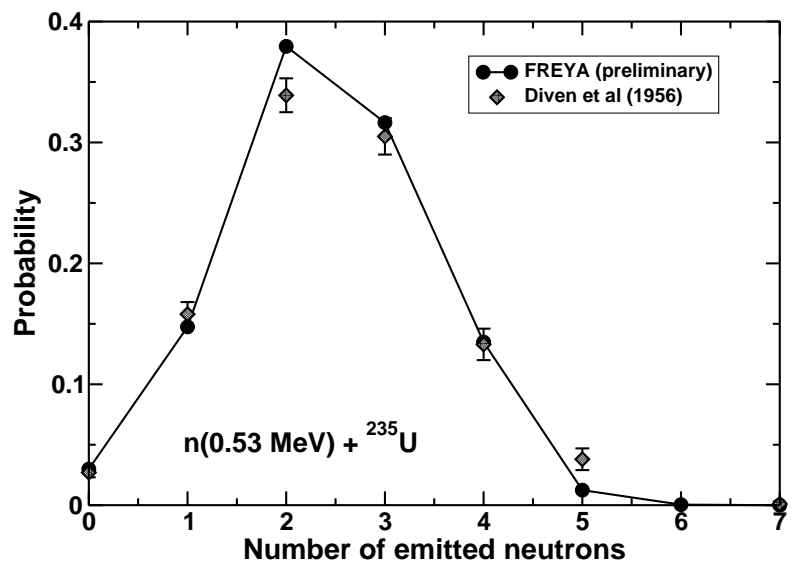

FIGURE 1. The normalized neutron multiplicity distribution obtained with FREYA compared to the data of Ref. [4].

Figure 2 and Table 1 show an example of the type of more exclusive observable that can be addressed with FREYA: the neutron kinetic energy spectra for a specified neutron multiplicity. We can consider either the multiplicity from both fragments combined or each fragment separately. The following features are apparent. Although the light and heavy fragments emit approximately the same number of neutrons on the average, the neutrons from the light fragment tend to be more energetic, as seen in the spectra of Fig. 2. The average energy of a neutron emitted by the light fragment is $2.02 \mathrm{MeV}$ while those from the heavy fragment have only $1.56 \mathrm{MeV}$. The average from both fragments combined is $1.80 \mathrm{MeV}$.

Furthermore, the average neutron energy decreases as the neutron multiplicity increases. This anticorrelation is expected because whenever a single neutron carries away most of the available energy any further emission of neutrons is disfavored.

We have also extracted the combined kinetic energy of the two fission products as a function of the average neutron multiplicity. Figure 3 shows the average values together with error bars that represent the dispersion in the kinetic energy from event to event. 


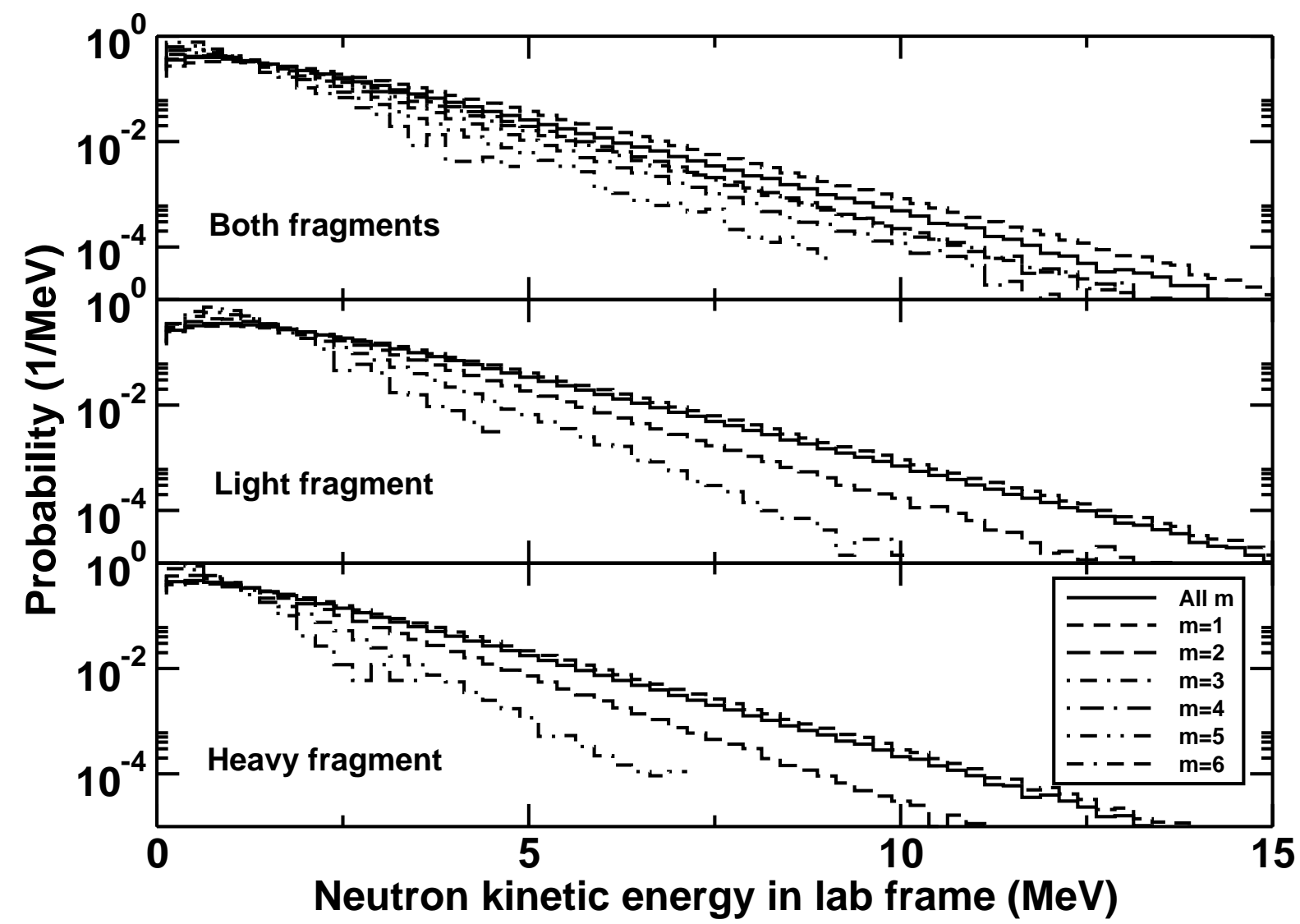

FIGURE 2. The emitted neutron kinetic energy spectra in the laboratory frame gated on a specified neutron multiplicity $m$, for either both fragments combined (top) or the light fragment (center) and heavy fragment (bottom) separately.

There is a pronounced anticorrelation between the kinetic energy and the multiplicity which is readily understood as a result of energy conservation. High neutron multiplicities arise primarily from hot, excited fragments which in turn are associated with low fragment kinetic energies.

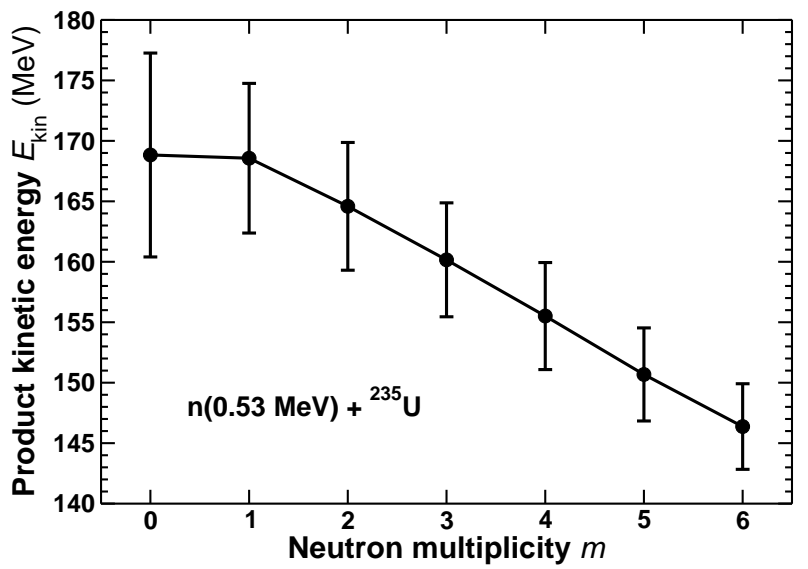

FIGURE 3. The combined kinetic energy of the two fission products as a function of the average neutron multiplicity.
In addition, it is apparent that the width of the kineticenergy distribution also decreases steadily with increasing neutron multiplicity, as one would expect. FREYA is also capable of addressing the corresponding correlation for just one of the fragments but this is less relevant since the source of a given neutron cannot be experimentally identified.

As a last illustration, we consider the angular correlation between two emitted neutrons. Since the average neutron multiplicity is not much larger than two, there is a good chance that any two neutrons detected in a given event arise from different fission fragments. These tend to move back-to-back due to momentum conservation and the associated opposite boosts of the neutron sources would be expected to introduce a tendency for the two neutrons to enter opposite hemispheres.

We have examined this effect by extracting the coincidence rate for two neutrons entering oppositely located solid angles characterized by a specified (common) opening angle $\theta$. The associated enhancement factor is given by this same-event coincidence rate divided by the corresponding mixed-event coincidence rate obtained by sampling the two neutrons from different events. 
TABLE 1. The mean laboratory kinetic energy, $\langle\varepsilon\rangle$, and the associated dispersion, $\sigma_{\varepsilon}$, of emitted neutrons in the reaction $0.53 \mathrm{MeV} n\left({ }^{235} \mathrm{U}^{235} \mathrm{U}^{*}\right) f$ for various neutron multiplicities, $m$.

\begin{tabular}{ccc}
\hline$m$ & $\langle\varepsilon\rangle(\mathrm{MeV})$ & $\sigma_{\varepsilon}(\mathrm{MeV})$ \\
\hline \multicolumn{3}{c}{ Both Fragments } \\
\hline all & 1.80 & 1.41 \\
1 & 2.10 & 1.56 \\
2 & 1.68 & 1.28 \\
3 & 1.54 & 1.25 \\
4 & 1.35 & 1.11 \\
5 & 1.18 & 0.95 \\
6 & 1.01 & 0.74 \\
7 & 0.99 & 0.53 \\
\hline \multicolumn{3}{c}{ Heavy Fragment } \\
\hline all & 1.56 & 1.25 \\
1 & 1.70 & 1.32 \\
2 & 1.25 & 1.01 \\
3 & 0.95 & 0.72 \\
4 & 0.75 & 0.53 \\
\hline \multicolumn{3}{c}{ Light Fragment } \\
\hline all & 2.02 & 1.73 \\
1 & 2.17 & 1.57 \\
2 & 1.73 & 1.23 \\
3 & 1.43 & 0.75 \\
4 & 1.21 & 0.71 \\
\hline \multicolumn{2}{c}{}
\end{tabular}

Figure 4 shows the resulting enhancement factor as a function of the opening angle $\theta$. As expected, the two neutrons exhibit a clear directional anticorrelation. Moreover, while the effect is only around ten per cent for $\theta=90^{\circ}$ (corresponding to two opposite hemispheres), it grows significantly larger as the angular acceptance is reduced and approaches two as $\theta \rightarrow 0$.

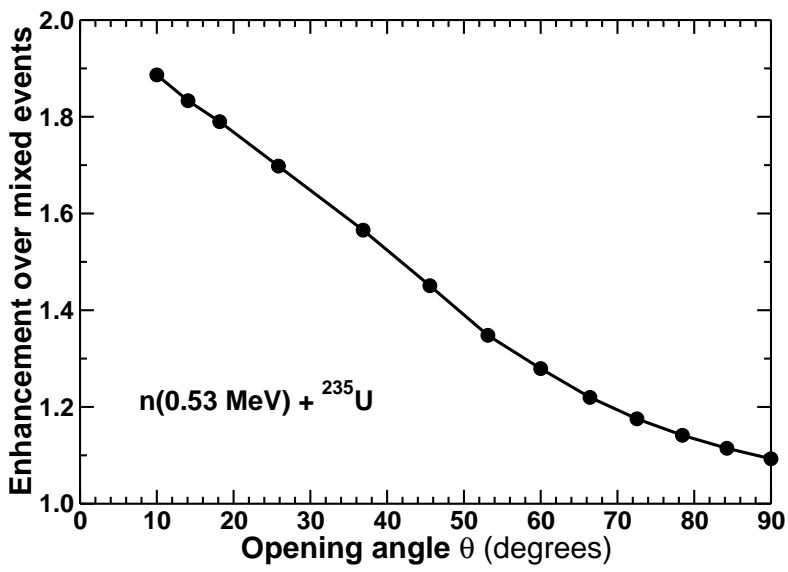

FIGURE 4. Enhancement factor for correlated neutron emission from two fission fragments relative to an uncorrelated mixed event background as a function of acceptance angle $\theta$.

\section{SUMMARY}

Whereas earlier numerical treatments of the decay processes associated with fission have tended to focus on average quantities, which are often sufficient, the next generation of treatments involves direct Monte Carlo simulation of individual event chains. In addition to our own work along these lines, a conceptually similar approach is being developed at Los Alamos by Talou [5, 6]. Such treatments give access to essentially any conceivable correlation observable and thus have vastly expanded potential utility. This undertaking presents challenges with regard to both physics input and programming technique.

On the physics side, the available information is insufficiently detailed to uniquely determine the treatment and a considerable degree of extrapolation to unknown territory must be made, based as far as possible on plausible physical ideas. Thee development work naturally identifies various kinds of new data that would be particularly helpful and the code may become especially useful in connection with the planned TPC fission detector. To facilitate the incorporation of known data, expected to be an ongoing process, the code has a modular structure.

A technical challenge consists in designing the code so that it will be sufficiently fast to be of practical use in transport studies where many different event samples must be generated. In its current manifestation, FREYA is quite fast, generating one million events in $12 \mathrm{~s}$ on a MacBook laptop computer.

\section{ACKNOWLEDGMENTS}

We thank D.A. Brown, T. Kawano, P. Möller, E.B. Norman, W.J. Swiatecki, P. Talou and W. Younes for discussions. The work of RV was performed under the auspices of the U.S. Department of Energy by Lawrence Livermore National Laboratory under Contract DE-AC5207NA27344 and was also supported in part by the National Science Foundation Grant NSF PHY-0555660. The work of JR was supported in part by the US Department of Energy, Contract Numbers DE-AC0205CH11231.

\section{REFERENCES}

1. M. Heffner, these proceedings.

2. W. Younes, these proceedings.

3. P. Möller, private communication.

4. B.C. Diven, H.C. Martin, R.F. Taschek, and J. Terrell, Phys. Rev. 101, 1012 (1956).

5. P. Talou, these proceedings.

6. S. Lemaire, P. Talou, T. Kawano, M.B. Chadwick, and D.G. Madland, Phys. Rev. C 72, 024601 (2005). 